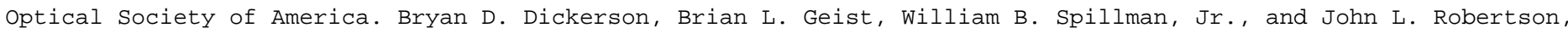

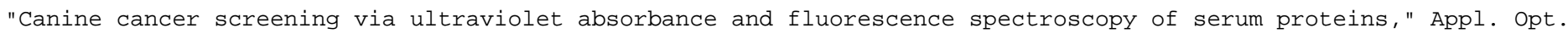
46 , 8080-8088 (2007). doi: 10.1364/ao.46.008080

\title{
Canine cancer screening via ultraviolet absorbance and fluorescence spectroscopy of serum proteins
}

\author{
Bryan D. Dickerson, ${ }^{1}$ Brian L. Geist, ${ }^{2, \star}$ William B. Spillman, Jr., ${ }^{3}$ and John L. Robertson ${ }^{3}$ \\ ${ }^{1}$ Luna Innovations Incorporated, 3157 State Street, Blacksburg, Virginia 24060, USA \\ 2Department of Physics, Virginia Polytechnic Institute and State University, Blacksburg, Virginia 24061, USA \\ ${ }^{3}$ Department of Biomedical Sciences and Pathobiology, Virginia Polytechnic Institute and State University, Blacksburg, \\ Virginia 24061, USA \\ *Corresponding author: bgeist@vt.edu
}

Received 22 March 2007; revised 30 September 2007; accepted 5 October 2007; posted 16 October 2007 (Doc. ID 81377); published 19 November 2007

\begin{abstract}
A cost-effective optical cancer screening and monitoring technique was demonstrated in a pilot study of canine serum samples and was patented for commercialization. Compared to conventional blood chemistry analysis methods, more accurate estimations of the concentrations of albumin, globulins, and hemoglobin in serum were obtained by fitting the near UV absorbance and photoluminescence spectra of diluted serum as a linear combination of component reference spectra. Tracking these serum proteins over the course of treatment helped to monitor patient immune response to carcinoma and therapy. For cancer screening, $70 \%$ of dogs with clinical presentation of cancer displayed suppressed serum hemoglobin levels (below $20 \mathrm{mg} / \mathrm{dL}$ ) in combination with atypical serum protein compositions, that is, albumin levels outside of a safe range (from 4 to $8 \mathrm{~g} / \mathrm{dL}$ ) and globulin levels above or below a more normal range (from 1.7 to $3.7 \mathrm{~g} / \mathrm{dL}$ ). Of the dogs that met these criteria, only $20 \%$ were given a false positive label by this cancer screening test. (C) 2007 Optical Society of America

OCIS codes: $170.1470,170.1610,170.3890,170.4580,170.6280,000.1430$.
\end{abstract}

\section{Introduction}

Nearly one fourth of all deaths in the United States are caused by cancer [1]. In 2004, over 500,000 Americans died from cancer. Early detection and treatment improves the odds of patient survival. Therefore, appropriate screening techniques are emphasized, such as regular mammograms for breast cancer. Given the carcinogenic risks of ionizing radiation, ultrasonic and optical techniques are desired as safer alternatives. Endoscopy can highlight tumors in the cervix, lungs $[2,3]$, and colon based on tissue autofluorescence and structure. However, these invasive tests are only suitable for cancer screening in high-risk populations. Encouraged by the success of several protein expression assays to detect specific carcinomas of the bladder and prostrate [4-6], oncologists have searched for a universal cancer marker. However, until a tumor of a certain size $(>1 \mathrm{~cm})$ or location is clinically detected,

0003-6935/07/338080-09\$15.00/0

(C) 2007 Optical Society of America there are very few general indicators of occult cancer. Consistent with the complex nature of cancer as a disease, advanced new technologies have been developed, such as hybrid forms of mass spectroscopy [6-8] and arrays of fluorescent probes conjugated with antibodies or DNA sequences [9], which enable researchers to search for patterns in protein expression in the hope of identifying malignancies.

There are good reasons to focus on blood composition for early detection of cancer. Healthy human serum consists mainly of albumin $(4.0 \pm 0.25 \mathrm{~g} / \mathrm{dL})$ and various globulins $(\sim 2.2 \mathrm{~g} / \mathrm{dL})$ in water [10]. Many forms of cancer disturb local vascular structure and trigger significant immune responses. Therefore, serum levels of globulins, albumin, and hemoglobin metabolites may be modified by the presence of various neoplasia.

Several studies have noted a downward shift from the normal human serum albumin:globulin ratio (1.33) as cancers progress. During acute inflammation, $\alpha$-globulins are released in response to cytokines, such as the tumor necrosis factor. Elevated $\beta$-globulin levels 
and monoclonal $\gamma$-globulin levels are often observed with multiple myeloma and lymphoma. An increase in C-reactive protein (a $\beta$-globulin) may occur as the body regulates inflammation in response to cancer. Chronic cancer conditions may also increase the levels of $\gamma$-globulins IgG, IgA, and IgM [11]. Using UV fluorescence spectroscopy, Hubmann et al. found higher serum levels of $\alpha_{2}$-globulins at the expense of albumin in patients with malignancies [12].

The relationship between serum albumin and cancer is less clear. Albumin synthesis in the liver increases $\sim 40 \%$ in cancer patients who experience a wasting of body mass, but cancer is often associated with low serum albumin levels. Changes in major protein levels (such as albumin and globulins) due to cancer seem to be more consistent with a whole-body response, rather than with local over expression of specific proteins by tumor cells [13].

If tumors induce hemorrhaging or weaken red blood cells walls, then hemoglobin would be released into the serum, either within the body or after blood is drawn from the patient. Because free hemoglobin quickly binds to blood proteins, it is usually not observed at meaningful levels in serum or plasma [14]. Instead, the levels of haptoglobin-hemoglobin complexes and some $\beta$-globulins increase when red blood cells break; and if hemolysis is severe, haptoglobin levels become depleted [11]. Therefore, to monitor total hemoglobin levels in serum, it would be useful to find a method that accounts for hemoglobin complexes as well. Furthermore, if high $\mathrm{pH}$ within tumors alters serum $\mathrm{pH}$, then the spectra of hemoglobin complexes (such as methemoglobin) may be modified [14]. Porphyrin can accumulate in tissue and serum if tumors alter iron metabolism [15-17]. The wellknown, strong autofluorescence of porphyrin has facilitated the visual detection of colon cancer and other malignancies, under UV light [18-20].

These biochemical interactions justify primary serum protein monitoring as a trial basis for cancer screening. However, traditional colorimetric techniques of measuring albumin, globulins, and hemoglobin in serum have limited accuracy, because the results can vary significantly depending on whether these proteins are initially in an isolated state or in the form of a protein complex $[21,22]$. When different facilities quote different normal physiological ranges for serum proteins, and give disclaimers about comparing results obtained from different laboratories, it is no wonder that some trials have not been able to detect recurring cancer with blood chemistry panels alone [23]. With advances in computers and spectrometers, there has been a recent renaissance in absorbance and fluorescence spectroscopy methods of quantifying albumin and total protein concentrations in serum, with improved sensitivity and linearity [21]. These developments facilitate affordable cancer screening.

Dogs are considered to be a good model of some types of human cancer and its response to therapy. Canine breast cancer has many parallels to human mammary carcinoma presentation [24]. Furthermore serum globulins and albumin levels in dogs are comparable with the levels in humans. Therefore, canine populations were chosen to test the feasibility of using UV serum spectroscopy for cancer screening. This paper presents a refined spectroscopic method of estimating the concentration of albumin, globulins, and hemoglobin compounds in canine serum, which enables sensitive monitoring of a patient's physiological response to illness and therapy and helps to identify patients with cancer.

\section{Methods}

From a random sample of 60 dogs brought in for veterinary attention, serum was collected from a network of veterinary clinics in Virginia's New River Valley. Each pet owner volunteered to participate in the study, and the attending veterinarian documented each animal's health on a questionnaire. Wolves, and dogs with renal failure, hyperthyroidism, epilepsy, or Addison's disease in the original ramdom sample were excluded from this cancer screening study, because the absorbance spectra from their serum appeared to be significantly different from those of other breeds of healthy dogs or dogs with cancer. Optical screening of serum for other diseases was viewed as an area for future research beyond the scope of this paper. Statistically, the resulting cancer screening study population included the following 47 serum samples. This study primarily focused on an optical comparison of serum proteins between a group of 21 healthy dogs and a group of ten dogs with different forms of cancer; both groups contained a mixture of breeds, ages, and genders. As a separate control group, eight geriatric dogs were also studied. In one dog with lymphoma, eight additional serum samples were taken over an eight-month observation period, and these were optically analyzed as an example of monitoring a patient's health status during treatment and disease progression. All relevant records available to the authors regarding treatment and health of this dog are summarized in Table 2.

All serum was drawn using standard vacutainers without anticoagulant. This allowed clotting to help separate red blood cells from the serum, and kept the extraction method uniform. Blood was centrifuged at low speeds to gently move the packed cell volume to the base of the tube with minimal rupture of red blood cells. Approximately $1 \mathrm{~mL}$ of serum was removed from the top of the tube and frozen for one to three years. About one hour before analysis, samples were thawed, gently mixed, and then diluted to $1 / 60$ of their original concentration by adding $40 \mu \mathrm{L}$ of serum to $2360 \mu \mathrm{L}$ of deionized water in a poly(methyl methacrylate) (PMMA) cuvette, with an optical path length of $1 \mathrm{~cm}$. This initial dilution generally kept the absorbance below 1.5 for wavelengths near $278 \mathrm{~nm}$. If the peak UV absorbance was higher than 1.5, the samples were diluted further. To maintain solution homogeneity, diluted samples were gently tipped sec- 
onds before performing optical spectroscopy or chemical analysis.

Fluorescence spectra of the diluted serum in PMMA cuvettes were acquired on a Hitachi F-4500 fluorescence spectrophotometer from 290 to $500 \mathrm{~nm}$ every $0.2 \mathrm{~nm}$, at a rate of $240 \mathrm{~nm} / \mathrm{min}$, with excitation at $280 \mathrm{~nm}, 2.5 \mathrm{~nm}$ slits, a $2 \mathrm{~s}$ integration time, and a photomultiplier tube bias of $700 \mathrm{~V}$.

Absorbance spectra were acquired on a Hitachi U-2001 UV-vis near-infrared (NIR) spectrophotometer with pure water in a PMMA cuvette as the reference sample. For rapid data acquisition, spectra were recorded every $1 \mathrm{~nm}$ from 265 to $500 \mathrm{~nm}$, at $400 \mathrm{~nm} / \mathrm{min}$, with medium detector response and a UV lamp change at $370 \mathrm{~nm}$. The slit width was $2 \mathrm{~nm}$. Every hour, the system response was rechecked by measuring the absorbance spectra of water; if the water's absorbance deviated from 0.000 by more than 0.01 near $400 \mathrm{~nm}$, then the baseline was redone.

For comparison, the Veterinary Teaching Hospital Clinical Laboratory Services of the Virginia-Maryland Regional College of Veterinary Medicine performed standard automated colorimetric blood chemistry analysis to monitor albumin, total protein, and hemoglobin in known serum phantom solutions and selected canine sera. To check for other possible sources of absorbance or scattering, bilirubin, cholesterol, and lipids were also measured in selected sera.

To compare the accuracy of spectroscopic and chemical analysis methods, known solutions of serum constituents were prepared from fresh powders, all purchased from Sigma-Aldrich (St. Louis, Missouri). Single-solute aqueous reference solutions were made at concentrations of $1 \mathrm{~g} / \mathrm{L}$ for canine albumin Cohn 5 (catalog number A9263); $1 \mathrm{~g} / \mathrm{L}$ for canine globulins, mainly $\alpha$ Cohn 4-1 (catalog number G7015); and $0.1 \mathrm{~g} / \mathrm{L}$ for human hemoglobin, including methemoglobin (catalog number H7379). Mixtures of albumin, globulins, and hemoglobin within physiological concentration ranges were made for chemical analysis and then diluted for spectroscopic analysis.

\section{Results}

On the average, serum from geriatric dogs and dogs with cancer tended to have higher absorbance from 265 to $500 \mathrm{~nm}$ than was seen in ordinary healthy dogs (Fig. 1). This early observation suggested that absorbance spectra might be useful for cancer screening; however, the large variability in serum absorbance within groups made it difficult to correlate a patient's health group with isolated spectral features.

Methodical spectral analysis was able to accurately estimate serum composition, because reference solutions of albumin, globulins, and hemoglobin had distinguishable absorbance features, as shown in Fig. 2. Although all three serum components had a peak near $278 \mathrm{~nm}$, hemoglobin also exhibited a peak near $406 \mathrm{~nm}$, globulins produced a significant "tail" that gradually decreased from 300 to $500 \mathrm{~nm}$, and albumin had no such tail or other peak. Likewise, the fluorescence spectra of these reference solutions were

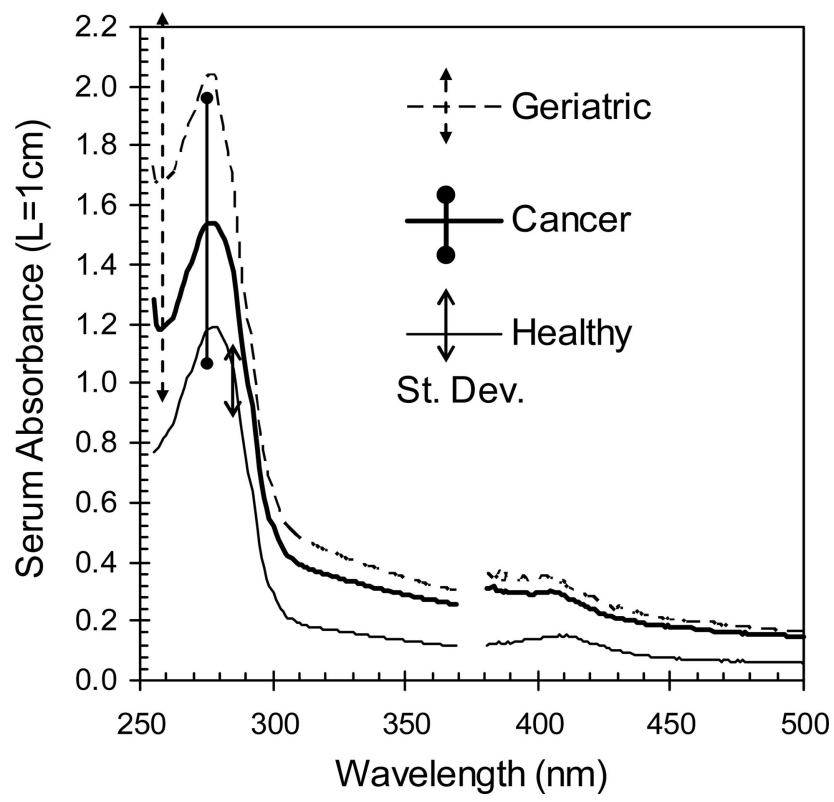

Fig. 1. Average UV absorbance spectra for diluted serum from dog populations grouped by health status. On the average, the serum absorbance of geriatric patients and those with cancer was higher than that of healthy dogs across the wavelength range from 250 to $500 \mathrm{~nm}$; however, there was high variability (standard deviations indicated by error bars) within the cancer and geriatric populations. The absorbance spectra shown are through samples that have been diluted in water to $1 / 60$ of their original concentrations.

also unique, as seen in Fig. 3. Hemoglobin emitted no significant fluorescence, nor did water or the PMMA cuvettes. Globulins produced bright narrow UV emission near $332 \mathrm{~nm}$, which was maximized using excitation at $280 \mathrm{~nm}$. Compared to globulins, albumin fluorescence intensity was lower near $332 \mathrm{~nm}$ at the same solute concentration $(1 \mathrm{~g} / \mathrm{L})$, and its peak was broader, so that albumin actually produced more emission near $300 \mathrm{~nm}$ than was seen from globulins.

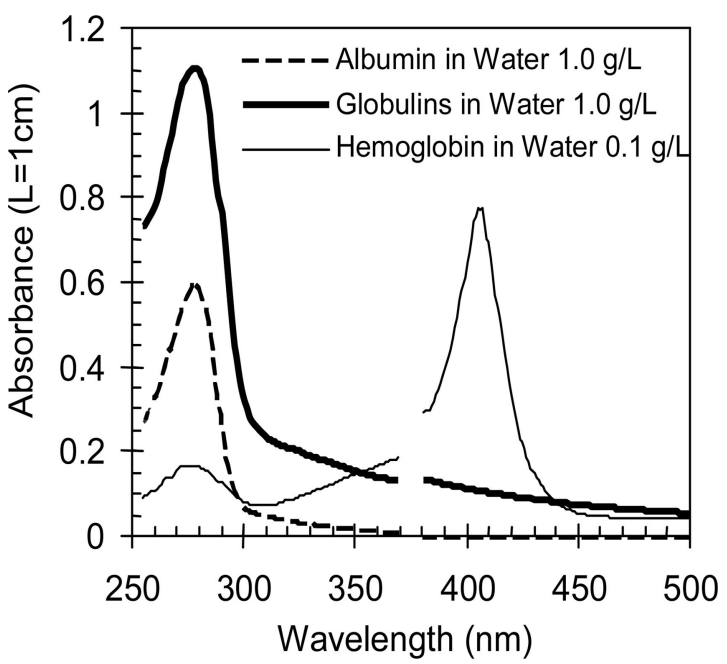

Fig. 2. Absorbance spectra of reference solutions for primary serum proteins. The spectral gap between 370 and $380 \mathrm{~nm}$ is a low signal region between two light sources. 


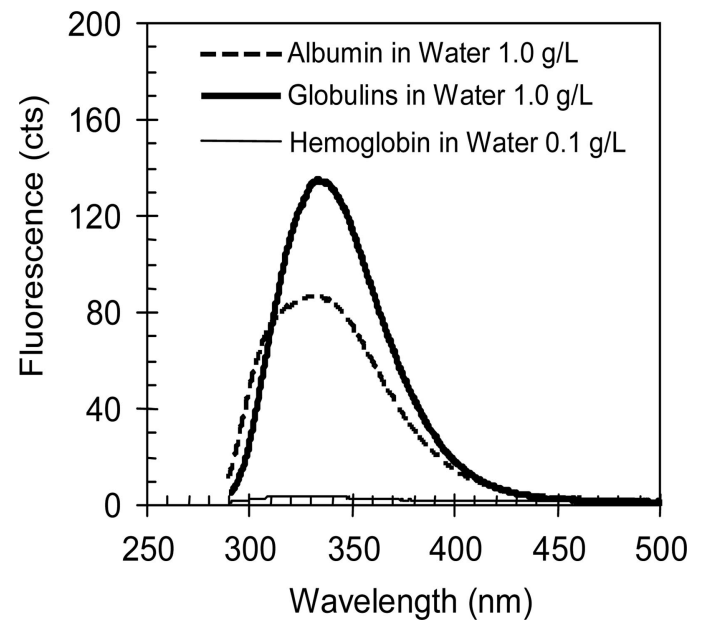

Fig. 3. Fluorescence spectra of reference solutions for primary serum proteins. Compared to albumin, globulins exhibited enhanced fluorescence near $300 \mathrm{~nm}$, while hemoglobin showed negligible fluorescence.

This difference in fluorescence peak shape provided additional information about the relative levels of albumin and globulins in fresh mixtures.

Absorbance peak amplitudes in Fig. 4 were proportional to solute concentration, as expected, so the observed absorbance spectra $A b s(\lambda)$ of known mixtures and diluted serum samples were modeled using Eq. (1) to generate a fit spectrum $A b s_{\text {fit }}(\lambda)$ from a linear combination of the reference spectra for albumin $A l b u(\lambda)$, globulins $G l o b(\lambda)$, and hemoglobin $\operatorname{Hem}(\lambda)$. The coefficients $A, G$, and $H$ represent the estimated concentrations (in $\mathrm{g} / \mathrm{L}$ ) of albumin, globulins, and hemoglobin in the diluted solution. $S$ represents the relative magnitude of a residual background contribution to the absorbance spectra (discussed below):

$$
\begin{aligned}
A b s_{\text {fit }}(\lambda)= & \frac{A}{1.0} \operatorname{Albu}(\lambda)+\frac{G}{1.0} \operatorname{Glob}(\lambda)+\frac{H}{0.1} \operatorname{Hem}(\lambda) \\
& +S \times \operatorname{Scat}(\lambda) .
\end{aligned}
$$

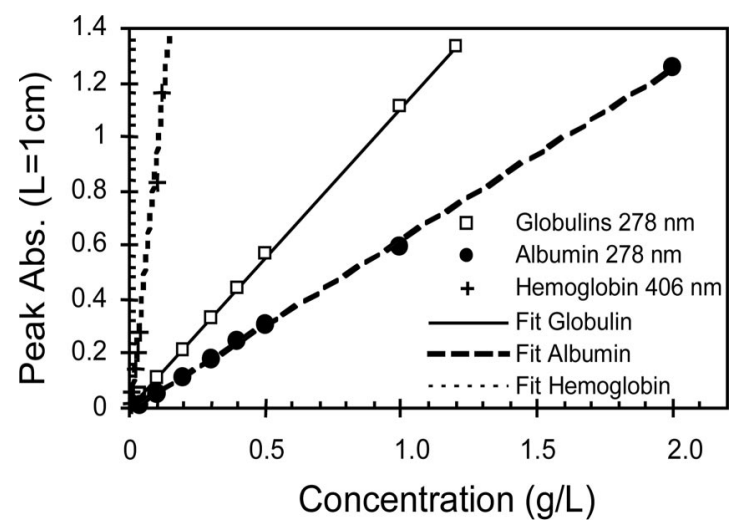

Fig. 4. Linear absorbance of serum components. The absorbance peak amplitude at the indicated wavelengths was proportional to that protein's concentration

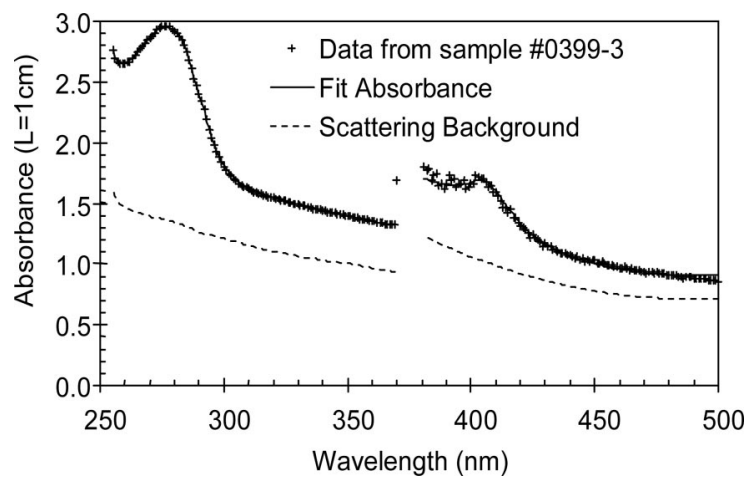

Fig. 5. Scattering contributions to serum absorbance spectra. The absorbance spectra of some samples exhibited a significant background, which increased towards shorter wavelengths. The dashed curve in this plot shows the smoothed scattering background, $\operatorname{Scat}(\lambda)$, after normalizing the spectra so that $\operatorname{Scat}(\lambda=$ $350 \mathrm{~nm})$ is 1 .

On several absorbance spectra with a turbid appearance, a high background was observed across the visible and UV region of the absorbance spectra, which could not be modeled as a linear combination of albumin, globulins, and hemoglobin. This background was assumed to originate primarily from scattering by turbidity, which would effectively reduce the transmitted intensity across a wide spectral range. To isolate the spectral shape of this component mathematically, the residual broad spectral contribution was calculated by subtracting albumin, globulins, and hemoglobin spectral features from the sample in Fig. 5, because it had a particularly high background. This background, $\operatorname{Scat}(\lambda)$, was smoothed and normalized so that it equaled 1.0 at $350 \mathrm{~nm}$, a convenient reference point where absorbance from other components was small. Canine serum spectra were then reanalyzed with this potential contribution. For those few sera where the fit was improved by including this background component, the globulin concentration estimates were reduced back to realistic physiological levels.

Multiparameter optimization was performed in Excel to estimate the values of the coefficients $A, G$, $H$, and $S$, which minimized the mean squared error $E_{a b s}$ between the observed and fit absorbance spectra as defined in Eq. (2), where $n$ is the number of wavelengths $\lambda$ in the spectra:

$$
E_{a b s} \equiv\left[\sum_{i=1}^{n}\left\{\left(\operatorname{Abs}\left(\lambda_{i}\right)-A b s_{\mathrm{fit}}\left(\lambda_{i}\right)\right)^{2}\right\}\right]^{1 / 2} / \sum_{i=1}^{n}\left\{\operatorname{Abs}\left(\lambda_{i}\right)\right\} .
$$

The fluorescence peak counts of albumin and globulin solutions at $332 \mathrm{~nm}\left(P F_{a l b u}\right.$ and $\left.P F_{g l o b}\right)$ increased asymptotically with concentration (in $\mathrm{g} / \mathrm{L}$ ) due to reabsorbance, as seen in Fig. 6 and characterized by Eq. (3). After dividing by each original peak height, the normalized fluorescence spectra $F_{\text {albu }}(\lambda)$ and $F_{\text {glob }}(\lambda)$ from these single solute solutions maintained their unique peak shapes for concentrations up to $\sim 1 \mathrm{~g} / \mathrm{L}$. 


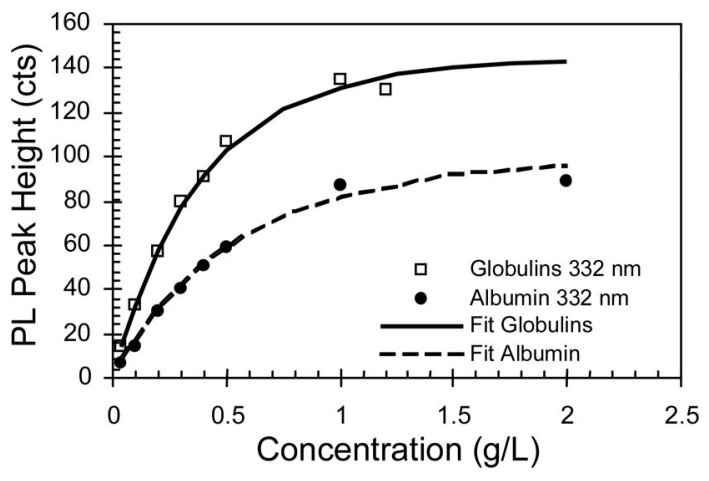

Fig. 6. Fluorescence of serum components saturates at higher concentrations due to reabsorbance.

Fluorescence spectra were modeled by scaling each normalized reference spectra by the calculated peak heights, as shown by Eq. (4), and the fit error was calculated using an expression analogous to Eq. (2):

$$
\begin{aligned}
P F_{a l b u} & =\frac{180 A}{1+1.2 A^{1.2}}, \quad P F_{\text {glob }}=\frac{368 G}{1+1.8 G^{1.2}}, \\
F_{\text {fit }}(\lambda) & =\frac{P F_{a l b u} F_{a l b u}(\lambda)+P F_{\text {glob }} F_{\text {glob }}(\lambda)}{P F_{\text {albu }}+P F_{\text {glob }}} .
\end{aligned}
$$

The diluted concentration estimates, $A, G, H$, and $S$ were varied using a standard multi-parameter optimization routine in Excel, in order to minimize the sum of the errors, $E_{a b s}+E_{f}$. This procedure was able to closely fit canine serum absorbance and fluorescence spectra as a linear combination of the component reference spectra, as shown in Fig. 7. In this method, the use of normalized fluorescence spectra effectively emphasized the absorbance spectra, because it scaled linearly with concentration. To calculate the original concentrations $A_{o}, G_{o}, H_{o}, S_{o}$, and $T_{o}$ of albumin, globulins, hemoglobin, scattering, and total protein, respectively, in the undiluted sample, the diluted concentration estimates were multiplied

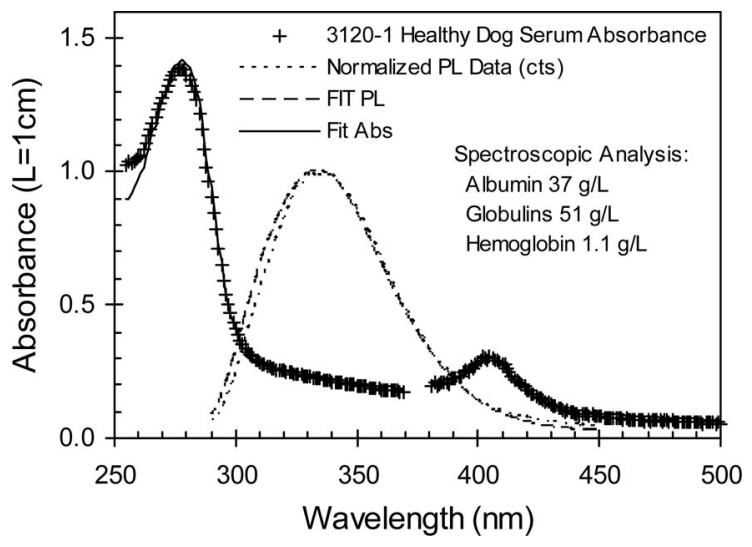

Fig. 7. Fitting typical serum spectra. Most all canine serum spectra were easily modeled as a linear combination of reference absorbance spectra and normalized fluorescence spectra from albumin, globulins, and hemoglobin.

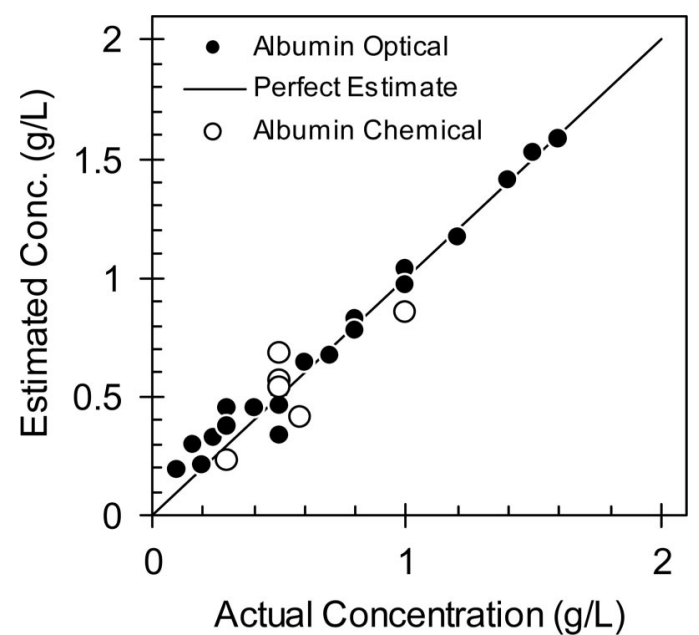

Fig. 8. Accurate spectroscopic estimates of albumin concentration in mixtures.

by the dilution ratio, $D . D$ was defined as the total volume divided by the small sample aliquot volume and was typically 60 . This method was calibrated against a series of known aqueous mixtures that simulated dilute serum.

UV spectroscopy was able to closely estimate albumin, globulins, total protein, and hemoglobin levels to within $10 \%$ of the actual levels in the multicomponent mixtures, as shown in Figs. 8-11. Compared to the spectroscopic method described above, standard chemical blood panel results were not as accurate, tended to underestimate globulins and total protein, and tended to overestimate hemoglobin levels. Such discrepancies in hemoglobin measurements are to be expected, since the standard blood panel methods were designed to characterize high concentrations of hemoglobin in whole blood, not very low concentrations in serum. In contrast, the spectroscopic method was a particularly sensitive and accurate method of

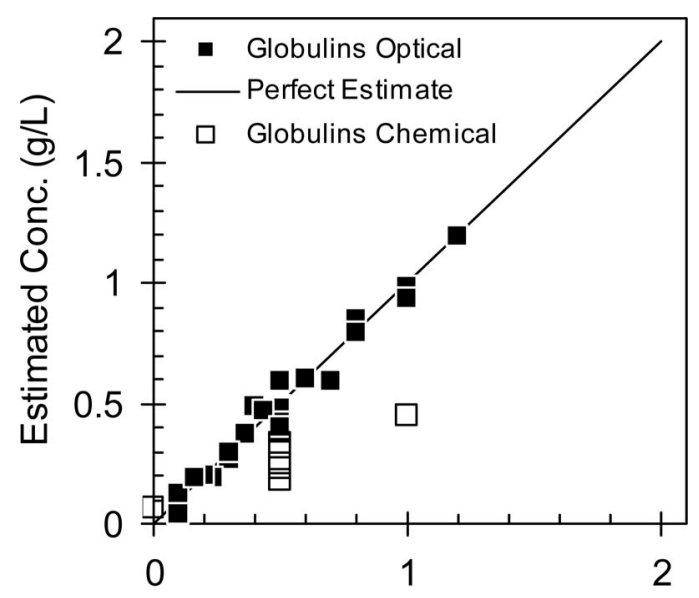

Actual Concentration (g/L)

Fig. 9. Accurate spectroscopic estimates of globulins in mixtures. Standard chemical blood tests (labeled "Globulins Chemical") underestimate globulin levels. 


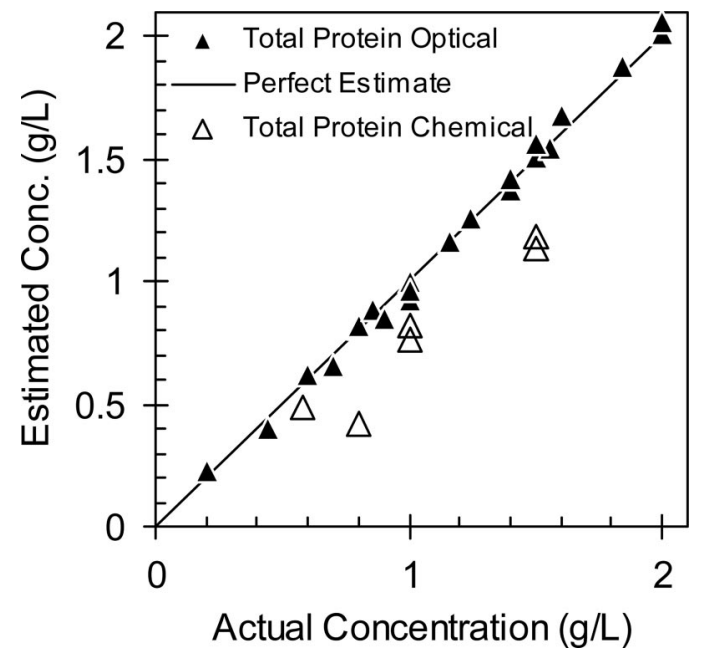

Fig. 10. Very accurate spectroscopic estimates of total protein (albumin + globulins) in mixtures, compared to less chemical test methods used in standard blood tests.

monitoring trace serum hemoglobin levels of less than $0.2 \mathrm{~g} / \mathrm{L}$ to within $10 \%$.

Accurate measurements of albumin, globulins, and hemoglobin levels in serum via the spectroscopic method enabled general cancer screening. When globulin levels were plotted versus albumin levels in Fig. 12, there was a set of coordinates with moderate albumin and globulin levels that described only healthy patients. Therefore, if the albumin level was between 40 and $80 \mathrm{~g} / \mathrm{L}$, and if the globulin level was simultaneously between 17 and $37 \mathrm{~g} / \mathrm{L}$, then the dog's blood parameters were labeled "safe." By plotting hemoglobin versus total protein (albumin plus globulin concentration) in Fig. 13, it was seen that most dogs with cancer had low serum hemoglobin levels. So, if the hemoglobin level was below $0.2 \mathrm{~g} / \mathrm{L}$, the serum parameters were labeled "suspect," as

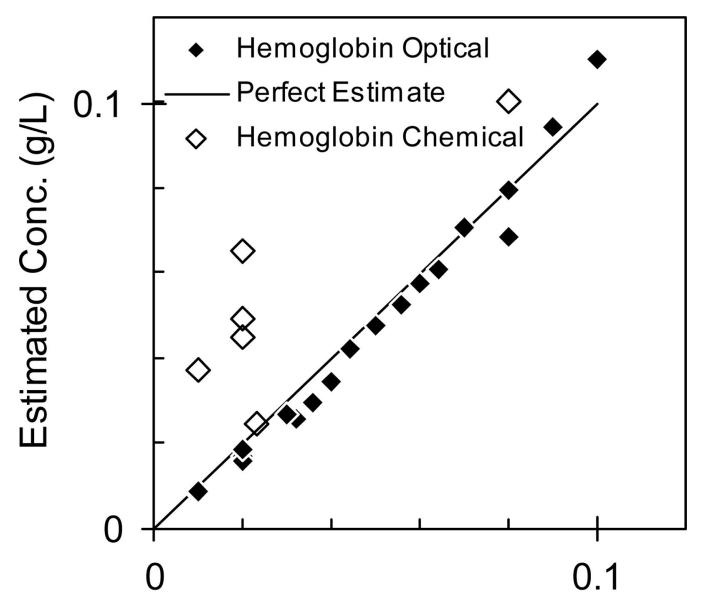

Actual Concentration ( $\mathrm{g} / \mathrm{L})$

Fig. 11. Sensitive spectroscopic estimates of trace hemoglobin levels in mixtures. Standard chemical blood tests (labeled "Hemoglobin Chemical") did not accurately measure such low hemoglobin levels.

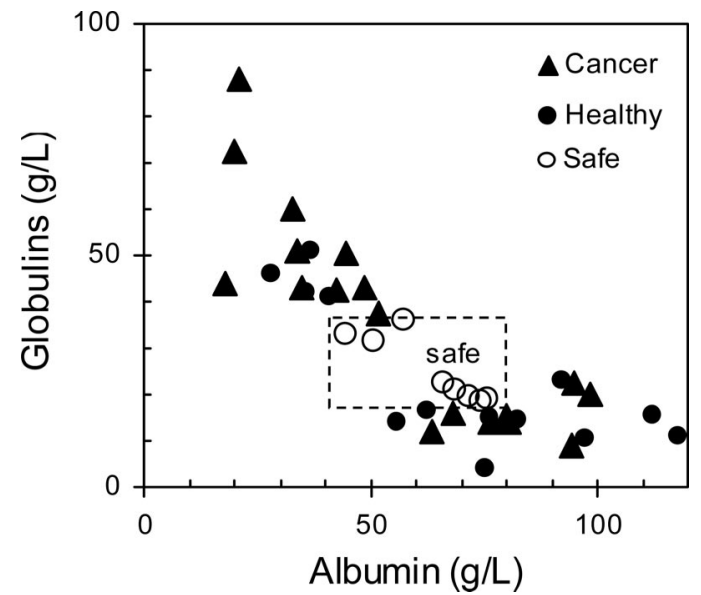

Fig. 12. Identifying selected low-risk patients based on globulins and albumin levels in serum. The "safe" subset of healthy patients could be excluded from the high-risk population based on their albumin and globulin levels, regardless of their serum hemoglobin and total protein levels.

shown in Fig. 13. If a dog's serum composition met the suspect criteria and did not meet the safe criteria, then further tests for cancer would be advised. Using these criteria, $\sim 70 \%$ of the dogs with cancer were correctly detected ( $30 \%$ false negatives), and $\sim 20 \%$ of those dogs flagged for further cancer testing would be expected not to show any clinical signs of cancer $(20 \%$ false positives). This set of criteria effectively reduces the probability of mistakenly flagging a healthy dog for further cancer tests.

\section{Discussion}

This spectroscopic method of analyzing serum exhibited several advantages over conventional photochemical blood panels. The spectroscopic protocol described above only required $40 \mu \mathrm{L}$ of blood serum to simultaneously measure albumin, globulins, and hemoglobin levels, with no consumable chemicals besides water. The blood chemistry laboratory, on the

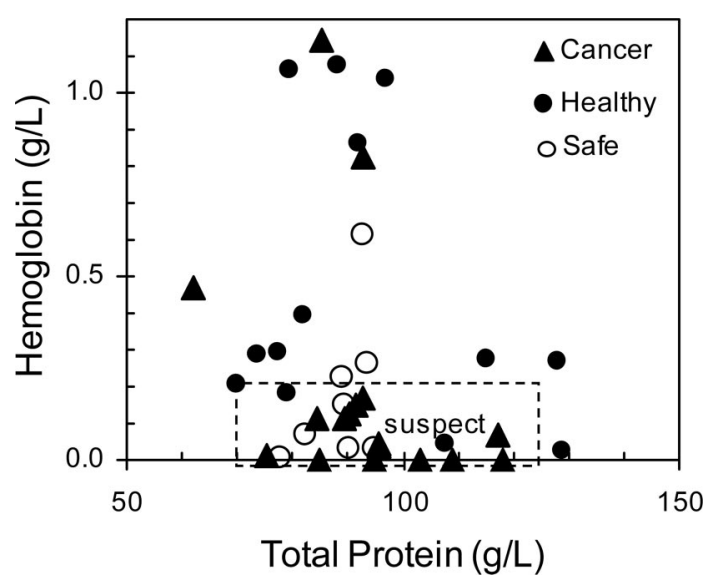

Fig. 13. Screening for cancer based on hemoglobin and total protein levels in serum. Most of the cancer patients had lower serum hemoglobin levels than the healthy patients, as shown in the "suspect" compositional region. 
other hand, required $400 \mu \mathrm{L}$, because each of these three constituents had to be analyzed in separate volumes with different photometric reagents. Compared to the conventional photochemical approach, the spectroscopic method is therefore inherently simpler to automate, while yielding more accurate results, especially in the determination of trace levels of hemoglobin in serum. These features facilitate the potential use of serum spectroscopy as a preliminary canine cancer screening tool.

The UV wavelength range from 250 to $500 \mathrm{~nm}$ was well-suited for rapid determination of canine albumin, globulins, and hemoglobin levels. Three common forms of hemoglobin listed below all have a strong peak near $410 \pm 5 \mathrm{~nm}$ [14]. Therefore, estimates of hemoglobin based on this peak were not affected by hemoglobin's oxidation state or its tendency to form protein complexes. The absorbance wavelength range could have been extended to $650 \mathrm{~nm}$ to include wellknown secondary peaks for hemoglobin in several forms: oxyhemoglobin at $576 \mathrm{~nm}$, methemoglobin at $630 \mathrm{~nm}$, or methemalbumin at $540 \mathrm{~nm}$ [14]. However, these peaks were not necessary to estimate the total hemoglobin concentration, and they were of negligible amplitude after the ideal 1/60 dilution for UV absorbance. It is possible that the chemical specificity of standard blood panels may limit their accuracy in estimating low hemoglobin levels in serum. For example, hemoglobin that is bound to albumin would not bind to the photometric reagent, and so the methemalbumin component would be underreported. Sensitive hemoglobin monitoring in serum may indirectly characterize red blood cell wall strength or frailty, if the applied stress from the blood drawing technique is kept constant.

The endogenous spectroscopy technique described here is limited, in that it does not detect trace amounts of several common serum pigments. Even when canine serum samples were only diluted to $1 / 4$ of their original concentration for absorbance near unity in visible wavelengths, none of the characteristic visible absorbance peaks were observed for carotenes, xanthophylls, porphyrins, or bilirubin. Instead, oxyhemoglobin features alone dominated canine serum absorbance spectra in the visible region. Using automated colorimetric techniques, bilirubin was generally found at normal physiological levels $(\sim 2 \mathrm{mg} / \mathrm{L})$ in selected canine serum samples by clinical laboratory services.

Rather than focusing on single marker proteins, this cancer screening technique uses patterns in the patient's albumin, globulins, and hemoglobin levels. Graphically, the approach shown in Figs. 12 and 13 is similar to principle component analysis, by which populations can sometimes be identified with clusters of data points when one basis vector of the parameter space variability is plotted versus another. However, the approach documented here has the intuitive benefit of physically defining what each principle component represents. In this study, the categorization of cancer samples was facilitated by simply plotting globulin levels versus albumin levels, and by plotting
Table 1. Correlation Coefficients between Canine Serum Protein Concentrations and Healthy Group ${ }^{a}$

\begin{tabular}{lrrrrrr}
\hline & $\mathrm{G}$ & $\mathrm{H}$ & $\mathrm{A}+\mathrm{G}$ & $\mathrm{A}$ & $\mathrm{A} / \mathrm{G}$ & $\mathrm{S}$ \\
\hline Globulins, $G$ & 1.00 & & & & & \\
Hemoglobin, $H$ & 0.09 & 1.00 & & & & \\
Total protein, & 0.06 & $\underline{-0.25}$ & 1.00 & & & \\
$\quad A+G$ & & & & & & \\
Albumin, $A$ & $\mathbf{- 0 . 7 7}$ & $\underline{-0.23}$ & $\mathbf{0 . 5 9}$ & 1.00 & & \\
Albumin:globulins & $\mathbf{- 0 . 7 4}$ & -0.04 & 0.18 & $\mathbf{0 . 7 1}$ & 1.00 & \\
$\quad$ ratio, $A / G$ & & & & & & \\
Scattering, $S$ & $\mathbf{0 . 3 4}$ & $\mathbf{0 . 7 2}$ & -0.13 & $-\mathbf{0 . 3 5}$ & -0.18 & 1.00 \\
Healthy group & $-\mathbf{0 . 3 6}$ & 0.06 & -0.18 & 0.17 & $\underline{0.23}$ & -0.22 \\
Cancer group & 0.17 & -0.01 & -0.14 & -0.23 & -0.15 & 0.15 \\
Geriatric group & $\underline{0.25}$ & -0.08 & $\mathbf{0 . 4 3}$ & 0.07 & -0.11 & 0.10 \\
\hline
\end{tabular}

${ }^{a}$ In this comparison of 47 samples, correlation coefficients that are larger than 0.28 are statistically significant (i.e., $p<0.05$ ), and these coefficients are shown in bold. Underlined coefficients are near this threshold, but the probability is greater than 5\% that the correlation between factors occurs just by chance, so such relationships are worth noting but with caution.

hemoglobin versus total protein. However, further studies with larger sample populations and perhaps more optically distinguishable blood components may enable the accuracy of cancer diagnosis to be improved by defining more complex mathematical relationships between the serum parameters.

It is understandable that healthy dogs in this study had moderate levels of globulins, whereas dogs with cancer tended to have either elevated globulins (suggesting in this case a systemic response to carcinoma) or reduced globulins (suggesting an immune suppressed state).

There seems also to be a compensating mechanism in dogs that works to keep the total protein level between 70 and $120 \mathrm{~g} / \mathrm{L}$. This hypothesis is supported by the clustering of data points along a diagonal line in Fig. 12, and by the strong negative correlation coefficient between globulins and albumin concentrations in Table 1.

With high sensitivity, this spectroscopic method was able to monitor a patient's response to therapy by tracking concentrations of free hemoglobin in serum as well as albumin and globulin levels. This technique could be widely used to optically monitor changes in a patient's health. It is well-known that the relative concentrations of albumin and globulins change with a patient's health status. In this case, a dog's response to therapy was optically monitored with the goal of tracking the progression or regression of cancer. Figure 14 shows how serum proteins and hemoglobin in a dog varied over the course of its treatment for cancer, which is outlined in Table 2. Reduced albumin levels were often associated with elevated globulin levels, while the total protein concentration remained fairly constant. In practice, such a test could be performed as part of a routine checkup, to establish a healthy baseline pattern. Then, personal deviations from one's own baseline would be clear. This would avoid the challenge of establishing general rules to account for 

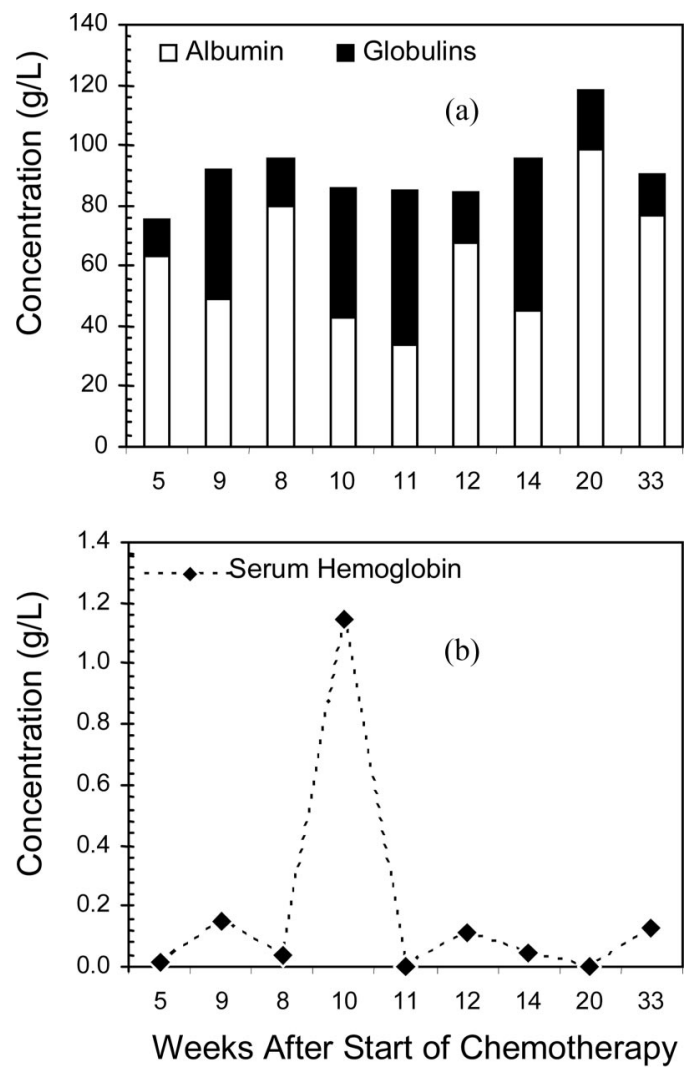

Fig. 14. Tracking temporal changes in serum composition for a dog with cancer. During the course of treatment following the Wisconsin protocol, fluctuations in (a) serum protein composition and (b) hemoglobin levels were observed using the optical methods described in this paper. The cause of the hemoglobin spike after 10 weeks of chemotherapy is unknown, but this dog's medical history is outlined in Table 2 .

many possible sources of serum compositional variation, such as age, diet, medical history, gender, heredity, etc. However, frequent testing of a patient may increase the effective cost of obtaining a diagnosis.

The normalized fluorescence spectra shape of fresh known serum phantoms was a helpful indicator of component protein levels. On the other hand, the fluorescence spectra of thawed dog serum in this study were considered less reliable, since they showed no significant spectral shape change, even when the absorbance spectra clearly indicated changes in the albumin to globulin ratio. Perhaps fluorescence is more sensitive than absorbance to serum aging. Further research is needed to asses the stability of serum proteins and their fluorescence spectra after years of frozen storage and after repeated freeze-thaw cycles.

It was hypothesized that the occasional presence of broadband absorbance was due to turbidity, perhaps from aggregated albumin complexes. This was suggested by the association of high scattering levels with reduced albumin levels in serum samples, as seen in the correlation coefficients listed in Table 1. The turbidity levels were highly variable and
Table 2. Medical History of a Dog Treated for Lymphoma

\begin{tabular}{cc}
\hline Week $^{a}$ & History \\
\hline-3 & A 5-year-old female (not neutered) Border Collie \\
& was first examined by Kirby at Companion \\
& Animal Clinic. Patient showed neck swelling and \\
& generalized lymphadenopathy. \\
& Treatment began with injection of chemotherapy \\
0 & following the Wisconsin protocol for 19 weeks. \\
3 & Right submand lymph node was ruptured. Analysis \\
& of a fine needle aspirate resulted in diagnosis of \\
& lymphoma with high-grade cell type. \\
8 & Viveristine was given. Despite cancer, overall \\
& health of the dog appeared good. \\
9 & Daxorubacin was given. \\
12 & Cytoxan was given. \\
19 & Tumor has generalized. \\
33 & Last treatment was given. \\
& Patient was out of remission for lymphoma, and \\
& health had deteriorated from good to fair. \\
& Observations included tumor presaps, pophtcals, \\
& submand, and enlarged lymph nodes.
\end{tabular}

${ }^{a}$ This is the number of weeks after the start of the Wisconsin protocol for chemotherapy. The corresponding results of sequential optical serum analysis are shown in Figs. 15(a) and 15(b).

were not significantly different in the serum of healthy and cancer populations.

To test and refine the initial cancer screening method and identification criteria presented here, further research is needed using a larger population of canine serum samples. The ultimate goal is to develop a preliminary cancer screening method for human serum. It is reasonable to expect that the spectroscopic deconvolution method, which was demonstrated for canine serum and serum phantom solutions, would also be able to estimate human serum albumin, globulins, and hemoglobin levels, possibly using a different ideal dilution ratio to keep the 278 $\mathrm{nm}$ absorbance peak amplitude near 1 . Of course, human and canine sera have different normal physiological ranges, so the boundaries of the safe and suspect serum parameter regions would need to be redefined based on statistical analysis of large welldefined patient populations.

\section{Conclusion}

A spectroscopic test method was developed for accurately determining the concentration of albumin, globulins, and hemoglobin in blood serum and for characterizing its turbidity. This test method compares measured near-UV absorbance and fluorescence spectra to a linear combination of reference protein spectra. The accuracy of this method exceeds that of standard blood chemistry panels and it detects trace levels of hemoglobin in any of its common chemical forms, while requiring a total of only $40 \mu \mathrm{L}$ of serum. This analytical method provided a sensitive way to track changes in serum composition over time to monitor the progression of cancer and to evaluate a patient's response to therapy. 
This technique formed the basis of an initial cancer screening test, that was demonstrated in a pilot study of 39 dogs. Using logical criteria involving the measured concentrations of albumin, globulins, and hemoglobin in serum, $70 \%$ of all patients with cancer were correctly identified as needing further tests; and only $20 \%$ of the flagged patients showed no clinical signs of cancer. Since dogs provide a good model of cancer development and effective therapy in humans, a similar approach may help identify humans with undetected cancer.

Canine serum samples were provided by Companion Animal Clinics. Preliminary spectroscopic analysis was performed by Nicholas Evans, Ian Civko, and Amr Sayedissa. Serum chemistry panels were run by Carolyn Sink. This research was funded by the Virginia Tech Applied Biosciences Center, and access to optical test facilities at the Fiber and Electro-Optical Research Center was provided by Richard O. Claus.

\section{References}

1. R. S. Cotran, V. Kumar, and T. Collins, eds., Robbins Pathological Basis of Disease (Saunders, 1999), pp. 260-327.

2. R. Richards-Kortum, R. Drezek, K. Basen-Engquist, S. B. Cantor, U. Utzinger, C. Brookner, and M. Follen, "Cervical dysplasia diagnosis with fluorescence spectroscopy," in Handbook of Biomedical Fluorescence, M. A. Mycek and B. W. Poque, eds. (Marcel Dekker, 2003), pp. 265-314.

3. G. Wagnières, A. McWilliams, and S. Lam, "Lung cancer imaging with fluorescence endoscopy," in Handbook of Biomedical Fluorescence, M. A. Mycek and B. W. Poque, eds. (Marcel Dekker, 2003), pp. 361-396.

4. E. P. Diamandis, "How are we going to discover new cancer biomarkers? A proteomic approach for bladder cancer," Clin. Chem. 50, 793-795 (2004).

5. S. C. Gad, ed., Drug Discovery Handbook (Wiley-Interscience, 2005), pp. 81-83.

6. K. P. H. Pritzker, "Cancer biomarkers: Easier said than done," Clin. Chem. 48, 1147-1150 (2002).

7. R. Aebersold and M. Mann, "Mass spectroscopy-based proteomics," Nature (London) 422, 198-207 (2003).

8. J. Li, Z. Zhang, J. Rosenzweig, Y. Y. Wang, and D. W. Chan, "Proteomics and bioinformatics approaches for identification of serum biomarkers to detect breast cancer," Clin. Chem. 48, 1296-1304 (2002).

9. A. Statnikov, C. Aliferis, I. Tsamardinos, D. Hardin, and S. Levy, "A comprehensive evaluation of multicategory classification methods for microarray gene expression cancer diagnosis," Bioinformatics 21, 631-643 (2005).
10. A. A. Pineda, ed., Selective Plasma Component Removal (Futura, 1984), p. 156.

11. B. F. Feldman, J. G. Zinkl, and N. C. Jain, eds., Schalm's Veterinary Hematology, 5th ed. (Lippincott Williams \& Wilkins, 2000), pp. 565-570 and 899-903.

12. M. R. Hubmann, M. J. P. Leiner, and R. J. Schaur, "Ultraviolet fluorescence of human sera: I. Sources of characteristic differences in ultraviolet fluorescence spectra from sera of normal and cancer-bearing patients," Clin. Chem. 36, 1880-1883 (1990).

13. S. Welle, Human Protein Metabolism (Springer, 1999), pp. 204-204.

14. R. J. Henry, D. C. Cannon, and J. W. Winkelman, eds., Clinical Chemistry Principles and Techniques, 2nd ed. (Harper \& Row, 1974), pp. 449, 1071-1072, 1117, and 1239.

15. M. Kondo, N. Hirota, T. Takaoka, and M. Kajiwara, "Heme biosynthetic enzyme activities and porphyrin accumulation in normal liver and hepatoma cell lines of rat," Cell. Biol. Toxins 9, 95-105 (1993).

16. R. Beri and R. Chandra, "Chemistry and biology of heme: effect of metal salts, organometals, and metalloproteins on heme synthesis and catabolism, with special reference to clinical implications and interactions with cytochrome P-450," Drug Metab. Rev. 25, 49-152 (1993).

17. B. R. Munson and R. J. Fiel, "A review: biochemical alterations associated with mouse spleen cells infected with Friend virus," J. Med. 4, 354-370 (1973).

18. T. S. Mang, C. McGinnis, C. Liebow, U. O. Nseyo, D. H. Cream, and T. J. Dougherty, "Fluorescence detection of tumors," Cancer 71, 269-276 (1993).

19. D. M. Harris and J. Werkhaven, "Endogenous porphyrin fluorescence in tumors," Lasers Surg. Med. 7, 467-472 (1987).

20. P. Jichlinski, M. Forrer, J. Mizeret, T. Glanzmann, D. Braichotte, G. Wagnières, G. Zimmer, L. Gulillou, F. Schmidlin, P. Graber, H. van den Bergh, and H. J. Leisinger, "Clinical evaluation of a method for detecting superficial transitional cell carcinoma of the bladder by light-induced fluorescence of protoporphyrin IX following topical application of 5-aminolevulinic acid," Lasers Surg. Med. 20, 402-408 (1997).

21. B. Leca-Bouvier and L. J. Blum, "Biosensors for protein detection: a review," Anal. Lett. 38, 1491-1517 (2005).

22. C. V. Sapan, R. L. Lundblad, and N. C. Price, "Colorimetric protein assay techniques," Biotechnol. Appl. Biochem. 29, 99108 (1999).

23. M. Weiss, C. L. Loprinzi, E. T. Creagan, R. J. Dalton, P. Novotny, and J. R. O'Fallon, "Utility of follow-up tests for detecting recurrent disease in patients with malignant melanomas," J. Am. Med. Assoc. 274, 1703-1705 (1995).

24. L. Pena, M. D. Perez-Alenza, A. Rodriguez-Bertos, and A. Nieto, "Canine inflammatory mammary carcinoma: histopathology, immunohistochemistry and clinical implications of 21 cases," Breast Cancer Res. Treat. 78, 141-148 (2003). 